\title{
Purification and Characterization of Carbon Nanotubes and the Formation of Magnetic Semiconductors for the Spintronic Application
}

\author{
Chernet Amente ${ }^{1}$, Keya Dharamvir ${ }^{2}$ \\ ${ }^{1}$ Physics Department, Addis Ababa Science and Technology University, Addis Ababa, Ethiopia \\ ${ }^{2}$ Physics Department, Panjab University, Chandigarh, India
}

Email address:

chernetamente@gmail.com (C. Amente),keya@pu.ac.in (K. Dharamvir)

\section{To cite this article:}

Chernet Amente, Keya Dharamvir. Purification and Characterization of Carbon Nanotubes and the Formation of Magnetic Semiconductors for the Spintronic Application. Science Research. Vol. 3, No. 3, 2015, pp. 122-128. doi: 10.11648/j.sr.20150303.22

\begin{abstract}
This article reports the synthesis of carbon nanotubes (CNTs) encapsulating iron (Fe) that would lead to formation of magnetic semiconductors, employing the arc discharge method. Morphology of the samples is studied from transmission electron microscope (TEM) imaging. Data is recorded by x-ray diffractometer (XRD) and identification of the sample constituent by energy dispersion x-ray (EDX). TEM images of metal added samples indicated that defects are completely removed after mono acidic treatment and open air oxidizing at $400^{\circ} \mathrm{C}$ for 15 minutes, leaving nano sized carbonaceous attached on surfaces of carbon nanotubes and catalyst particles encapsulated. This formation is recognized as a phenomenon at certain temperature. EDX examination shows that there is oxygen constituent remaining after purification along with iron and carbon, perhaps forming $\mathrm{FeO}$ during the reaction with water, indicating success in metal incorporation. This envisages that there would be formation of magnetic semiconductors where iron ions may take carbon cites in the CNTs of semiconducting characteristics, as can be revealed from experiments. This suggests that magnetic carbon nanotubes can be used for the spintronic application.
\end{abstract}

Keywords: Arc Discharge, Carbon Nanotubes, Defects, FeO, Magnetic Semiconductors

\section{Introduction}

Carbon nanotubes are a front line research topic since last two decades [1, 2]. Their discovery was in electric arcdischarge experiments for fullerene synthesis [1]. Carbon Nanotubes, CNTs, have been produced mainly by various methods involving gas solid reactions such as arc discharge, laser ablation, catalytic chemical vapor deposition (CVD) and plasma assisted deposition [3].

The quality and quantity of the nanotubes is understood as to depend on the type of the discharge method, the environment, annealing temperature, annealing duration, refluxing temperature, refluxing duration, system geometry, the electric current and voltage applied, and type of acids used for the reflux [4-6]. Experimental works have been reporting that these tubes can be of either with metallic [7] or semiconducting character $[8,9]$.

Theoretical electronicband structure calculations have also predicted that the chirality $(n, m)$ indices and diameter determine whether a SWNT has a metallic or semiconducting behavior $[10,11]$.
Because of their unique properties, CNTs are attractive materials for a wide range of applications such as biosensors $[12,13]$, fillers [14], gas and energy storage [15], efficient source of electron field emitters [16], that enabled fabrication of field-effect transistors based on individual single- and multi-wall with analyzed performance $[8,17,18]$, chargeable batteries [19], and semiconductor devices [20]. Moreover, for their gate effect narrow diameter SWCNTs are required for applications in carbon nanotube-based field effect transistors [8] than larger diameter MWNTS in some semiconductor devices, where band gap of semiconducting CNTs is understood to decrease with increasing diameter [19]. Thisnovel functionality can bring dynamism combined with magnetic ions such as iron, nickel and chromium integratingsemiconductivity and storage facilities in a single crystal.

The effect of metal added CNTs was studied systematically by plasma-enhanced hot-filament chemical vapor deposition and known to determine the diameter of the 
nanotube diameter [21]. Iron catalyzed production of CNTs have been investigated using different incorporation mechanisms and technique $[6,22]$ for both quantity and quality improvement.

In the present work, samples are prepared with the arc discharge method in a de-ionized water environment from low cost and locally devisable benefits point of view. Iron incorporation was done through powder mixing in which characterization is carried out in two phases. Pre- and post purification using various techniques.

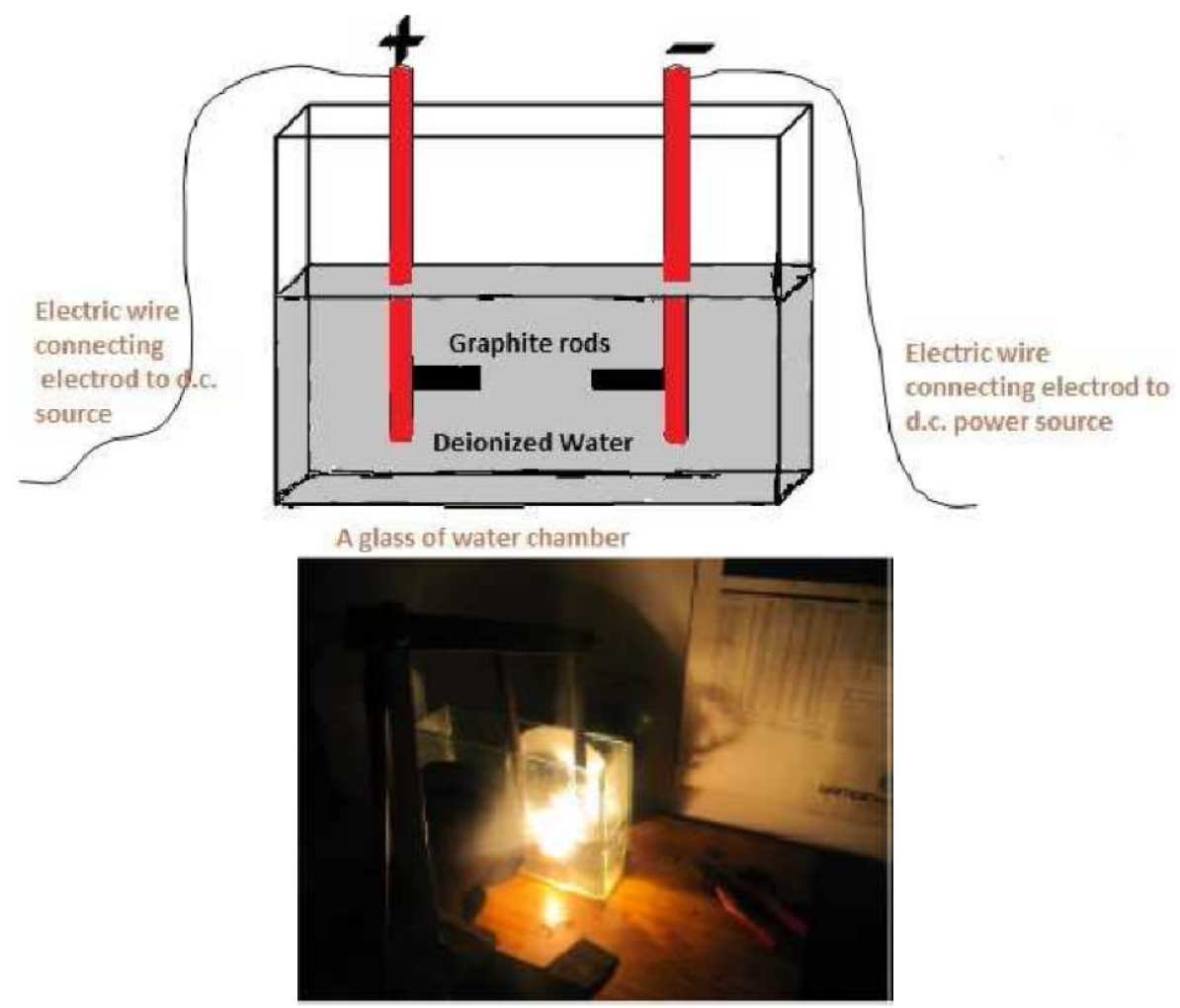

Fig. 1. The schematic diagram of the arcing equipment locally devised [23].

\section{Experimental}

In sample preparation we used carbon graphite rods commercially available. These rods were cut into pieces and drilled from one end forming a well structure. A mix of graphite and iron powder of 1:1 molar ratio is stuffed in to each well and mounted horizontally on the electrodes of the arc used as anode and cathode, few millimeters apart in a chamber of deionized water cover, as shown in fig.1, before the process [23].

A d.c of 50-200Adriven by a 40 Vpotential creating a high temperature discharge between the electrodes was applied. After frequent arcing, soot is formed in the water. The soot of water is then transferred into a bigger beaker after the removal of the chamber from the setup. Six hours later, the surface water was decanted and the remaining crude made dry in an oven for about 12 hours at $100^{\circ} \mathrm{C}$. Finally, the sample was tested for the purity by powder $\mathrm{x}$-ray diffractometer, XRD Cu-K $\alpha_{1}$, X'Pert PRO PANalytical.

The CNTs collected in the form of soot were purified by refluxing in a strong oxidant $8 \mathrm{M}$ Nitric acid $\left(\mathrm{HNO}_{3}\right)$ for the removal of catalyst metal for 24 hours. Subsequently, filtration with a $0.25 \mu$ mfilter membrane with the aid of a pump and thoroughly washing with distilled water until the
$\mathrm{pH}$ value reaches neutral was done. Finally, the samples were dried in an oven at $100^{\circ}$ cfollowed by open air oxidizing for $15 \mathrm{~min}$ at $400^{\circ} \mathrm{Cin}$ a furnace for the removal of impurities/amorphous carbon and other unreacted reagents $[6$, 24]. Ultimately, the product was analyzed by XRD, TEM, HR-TEM, and EDX.

\section{Results and Discussion}

The XRD measurement of the pristine sample shows that there are no apparent peaks attributed as introduced by defects, as can be seen from fig.2a. However, the XRD plot of the as prepared sample from iron doping in the $\mathrm{Fe}: \mathrm{C}$ 1:22 molar ratio prepared from previously purchased graphite rods of the same company indicated that impurities can be introduced industrially during preparation of the sample source. This was revealed from fig. 3 and other reports [25] directing to preferably use the later with increased molar ratio $(1: 1)$ in order to prevent degradation especially on mass production. This suggests that quality of the source material can persuade the purification rate as well.

The XRD measurement data plotting of purified sample shown in fig. $2 b$ indicates that there are few remnant iron nanoparticles after purification and the product is of carbon 
content attributed as of CNTs at large, as can also be seen from table I. The relatively small diffraction peaks associated with $\mathrm{Fe}$ phase suggested the presence of well crystallized iron grains with certain amount of size [26]. Peak positions in both panels of fig. 2 are the same except that there are slight shifts in which those of graphite and Fe are dominating, and perhaps some of them might have been overwhelmed by noise before purification
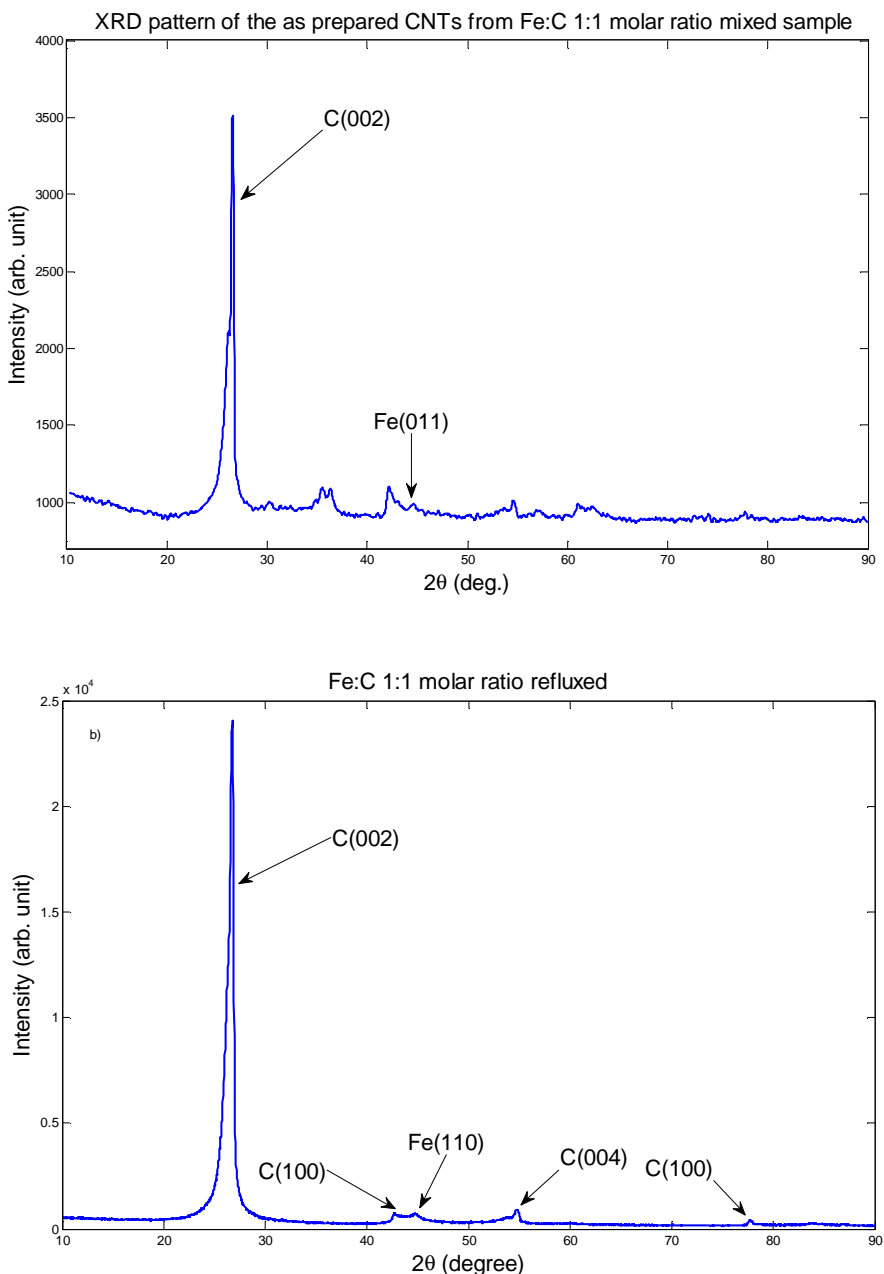

Fig. 2. XRD pattern of CNTs from Fe:C1:1 molar ratio as prepared (a) and post purification (b) with their corresponding planes [25].

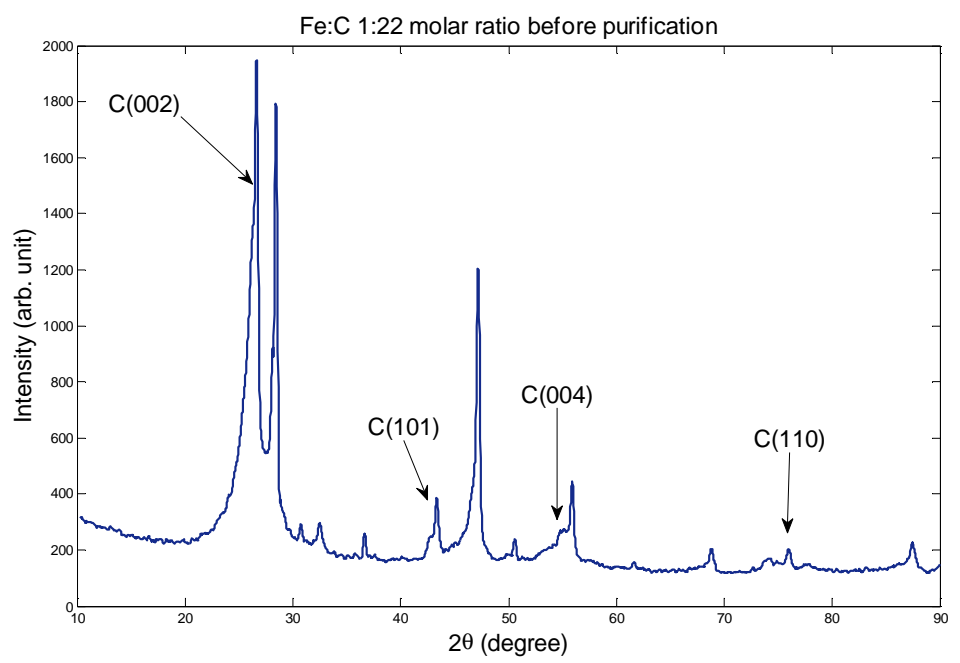

Fig. 3. XRD pattern of as prepared CNTS from C:Fe 22:1. The diffraction peaks emerged at $26.42^{\circ}$ corresponds to C(002) plane, 43.43 corresponds to C(101) and so on have resemblance with that of graphite. The remaining peaks reveal the existence of any other kind of ingredient including iron nano particles in the sample. 
In this study, the TEM images where collected after sonication of the samples for 5 days suspended in ethanol using a device of power $2 \mathrm{keVmodel}$ HITACHI 7500, maximum magnification of $6 \times 10^{6}$ times and resolution $0.2 \mathrm{~A}^{0}$

According to fig. 4 most of the CNTs have cone shaped tips in agreement with previous results [1], and also cut slantly from one end. Those open at the middle along their length are found in bundle with some roped creating graphitic nano-ribbons and also layered over one another (as in fig. 4a and c), as for the case of SWNTs [28]. There are others with striped surfaces closed from both ends standing separately (as shown in fig. 4d). Gloomy spot like particles (shown in fig. $4 \mathrm{a}, \mathrm{b}$ and c) are observed on the surface of the tubes as well. There are bean shaped nano-rings of inner diameter $5 \mathrm{~nm}-8 \mathrm{nmwith}$ thickness 3 nmlocated scattered elsewhere on the surface of graphene sheets and also attached to sides of some of the CNTs, oriented parallel and perpendicularly, as in fig. 4b. This situation is confirmed by high resolution transmission electron microscopy (HR-TEM) images, detected by apparatus operating at $200 \mathrm{keV}$ accelerating voltage, and shown as in fig. 5b. Speculations from further scrutiny indicate that the surface attached systems are CNTs of hexagonal structure with multilayered rings, as shown in fig. 5a too.
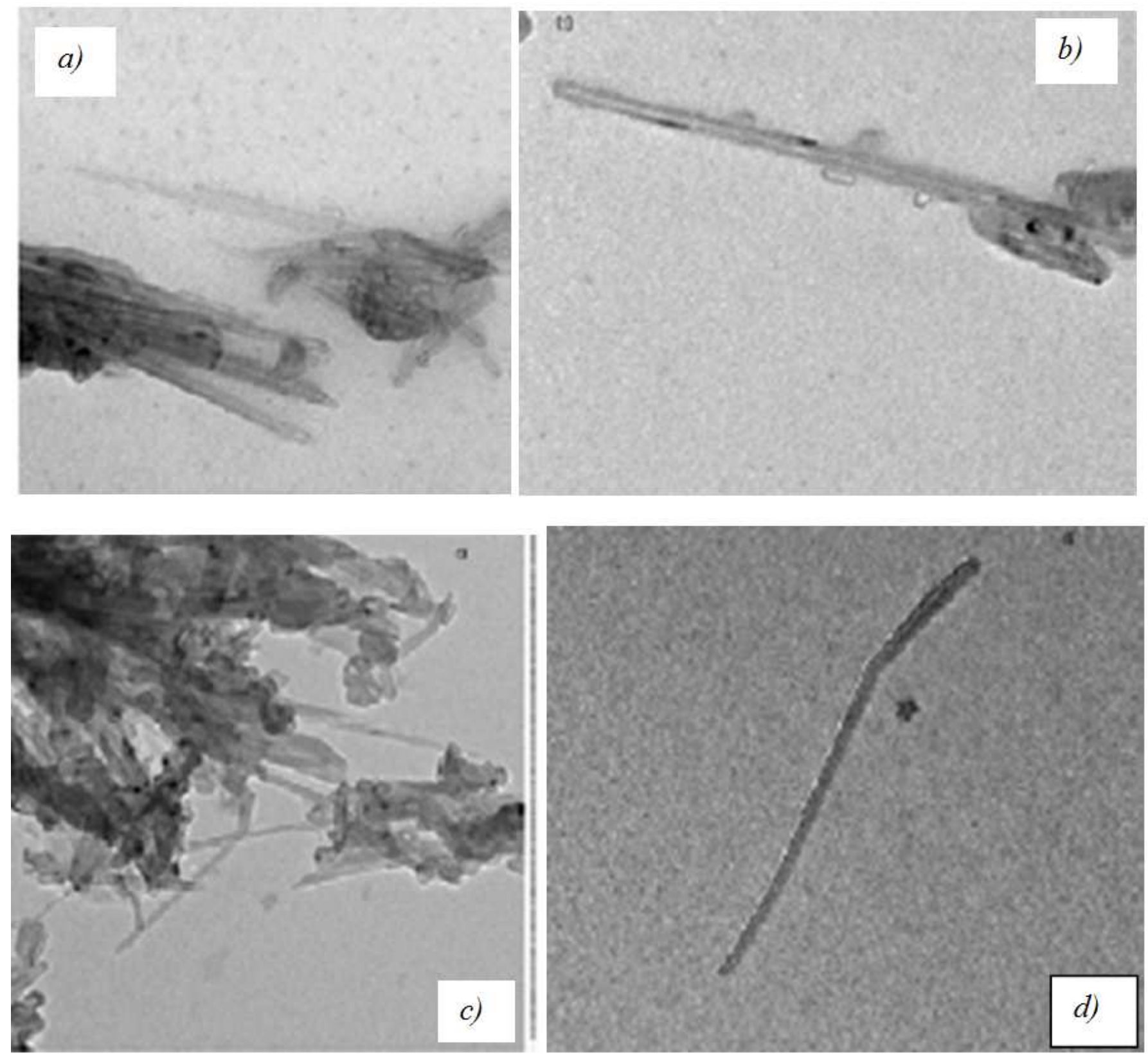

Fig. 4. TEM images of CNTs prepared from $\mathrm{Fe}: \mathrm{C}$ 1:1 molar ratio, after purification.

Both fig. 4 and fig. 5 reveals that there are CNTs longitudinally cut open from center and some observed to 
have multilayered structure and parallel to the axial direction exhibiting a good crystallinity. Fig. 5b shows the 3D scanning transmission electron microscopy, STEM structure of graphene sheet with attachment of CNTs and fullerene on the surface. Moreover, thepresence of $\mathrm{Fe}$ in the sample and acidic treatment is understood as increasing mass production of CNTs in comparison to their absence at the temperature limit the experiment is carried out, perhaps reducing breakages [25].
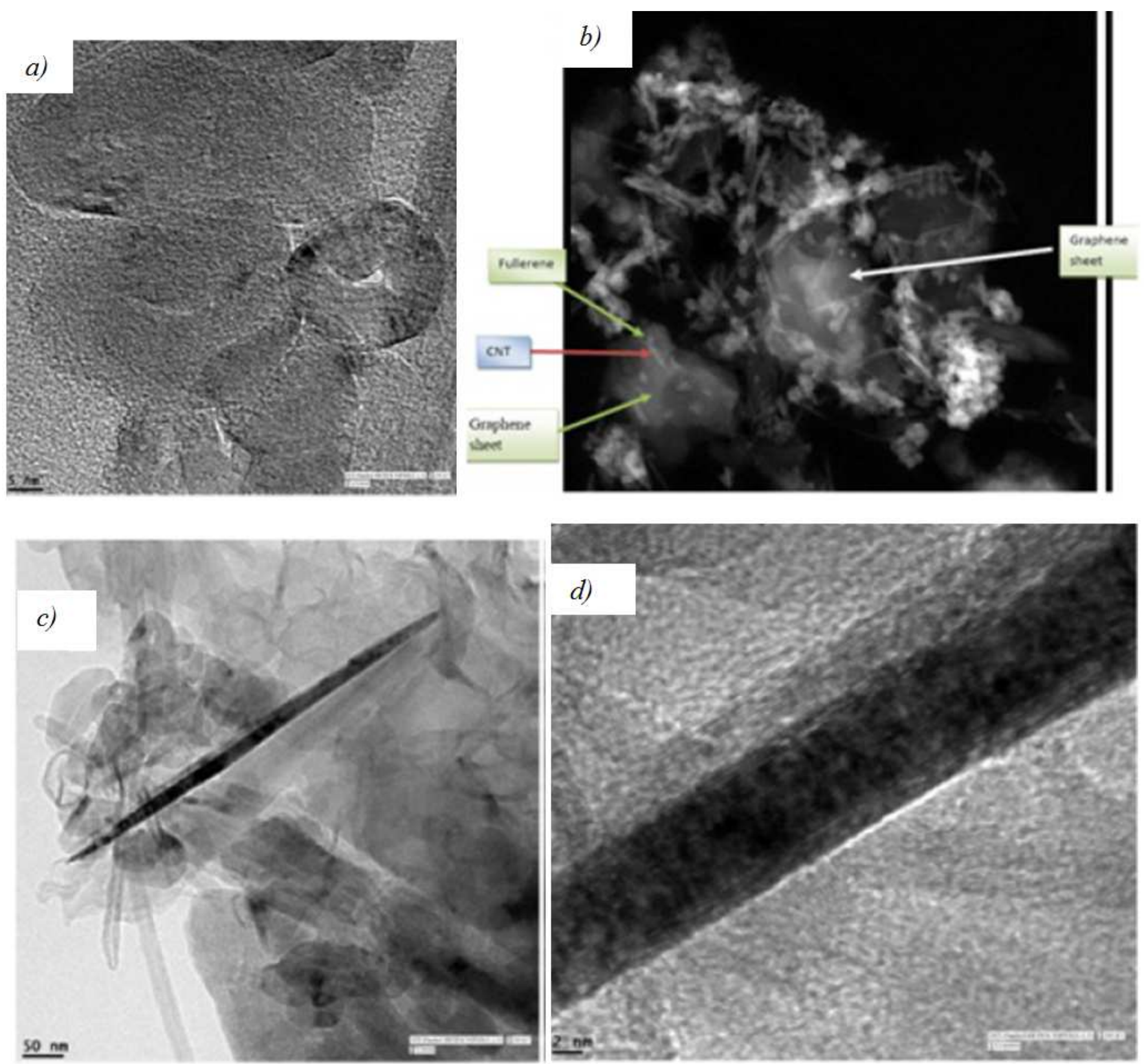

Fig. 5. HR-TEM and STEM images of CNTs prepared from Fe:C 1:1 molar ratio after purification a) HR-TEM images of high magnification of the nanorings/fullerene attached to each other b) STEM 3-D images of graphene sheet c) distribution of CNTs and fullerene on the surface of graphene sheet d) magnified form of panel $c$, showing morphology of CNT filled with iron particles.

Incorporation of iron nano particles into CNTs from inside is illustrated, as in fig. $5 \mathrm{c}$ and $\mathrm{d}$, indicating such carbon nanotube composite can be engineered for the purpose of data storage beside optical and transport facilities. Perhaps, the merging could follow substitutional or interstitial scheme where the later could degrade ferromagnetism. The substitutional method may well assist introduction of magnetic spins, leading the semiconductor CNTs to acquire magnetic semiconductor property. In this situation carbon of four valence electrons could be replaced by iron of two valence electrons with six localized $d$ sub shell electrons $\left(3 d^{6}\right)$ forming $\mathrm{sp}^{3}$ hybridization. This would introduce holes for ferromagnetic mediation of the localized spins, following Dietl model [29], establishing exchange energy, explained by the well-known Heisenberg mathematical expression $\mathrm{H}=$ $\sum_{\mathrm{ij}} \mathrm{J}_{\mathrm{ij}} \mathrm{S}_{\mathrm{i}} \mathrm{S}_{\mathrm{j}}$ where $\mathrm{J}_{\mathrm{ij}}$ is exchange energy of magnetic spins $\mathrm{S}$ localized at cites $\mathrm{i}$ and $\mathrm{j}$. This can also ascertain a new scheme and a useful motivation for systematic study of 
properties of carbon nanotube coated iron in analogy with the gold coating iron nano clusters, aiming at understanding the magnetic properties of core-shell structure used in biomedical applications [30].

According to Energy dispersive X-ray spectroscopy (EDS) analysis, weight percentage content of the iron particles in the purified product is about 0.29 , as also can be seen from Table I where copper, $\mathrm{Cu}$ is introduced due to copper grip on which the sample was deposited.

Table I. Energy dispersive X-ray spectroscopy (EDX) elemental analysis of the sample.

\begin{tabular}{llllll}
\hline $\begin{array}{l}\text { Quantification Results } \\
\text { Correction method: None }\end{array}$ & \multicolumn{1}{l}{} \\
\hline elements & Weight \% & Atomic \% & Uncert. \% & Detector correction & K-factor \\
$\mathrm{C}(\mathrm{K})$ & 85.92 & 95.72 & 0.61 & 0.26 & 3.940 \\
$\mathrm{O}(\mathrm{K})$ & 2.08 & 1.74 & 0.08 & 0.49 & 1.974 \\
$\mathrm{Fe}(\mathrm{K})$ & 0.26 & 0.06 & 0.02 & 0.99 & 1.403 \\
$\mathrm{Cu}(\mathrm{K})$ & 11.72 & 2.46 & 0.14 & 0.99 & 1.667 \\
\hline
\end{tabular}

\section{Conclusions}

Preparation of CNTs in de-ionized water and purification through acidic treatment is done successfully. Their morphology is studied applying the transmission electron microscopy and elemental analysis by the x-ray diffractometer. Production of Fullerene is identified as a Phenomenon of attachment on the surface of CNTs at $400^{\circ} \mathrm{C}$, the maximum annealing temperature limit considered in this experiment. Iron encapsulation is done by powder mixing mechanism at Fe:C 1:1 molar ratio. After acidic treatment the remaining product is found to be more of CNTs, fullerene, few graphene sheets and other carbonaceous impurities. According to the TEM images most of the CNTs have cone shaped tips and cut slantly from one end. Those open at the middle along their length are found in bundle with some roped and layered over one another. The XRD and EDX analysis show that there are carbon, oxygen and iron contents remaining in the sample after purification, perhaps forming $\mathrm{FeO}$ as well during the reaction, indicating that iron is successfully incorporated. Such carbon nanotube composite can be engineered for the purpose of data storage beside optical and transport facilities.

\section{Acknowledgements}

We would like to thank Dr. Charanjit Singh, Chemistry Department Panjab University, for his assistance during laboratory activities. We also acknowledge the financial support from the C. V. Raman fellow ship for African researchers.

\section{References}

[1] S. Iijima, "Helical microtubules of graphitic carbon,"Nature, vol. 354, pp. 56-58, 1991.

[2] S.Iijima, andT. Ichihashi, "Single-shell nanotubes of 1 - nm diameter,"Naturevol. 363, pp. 603-605, 1993

[3] C. Journet,and P. Bernier, "Production of carbon nanotubes," Appl. Phys.,vol. A 67, pp. 1-9 1998.
[4] A. Yoshinori, Z. Xinluo, I. Sakae, S. Iijimaa, "Mass production of multiwalled carbon nanotubes by hydrogen arc discharge,"J. Crys. Growth, vol. 237-239, pp.1926-1930, 2002.

[5] Z. Shi, Y. Lian, X. Zhou, Z. Gu, Y. Zhang, S. Iijima, L. Zhou, T. K. Yue, and S. Zhang, "Massproduction of single-wall carbon nanotubes by arc discharge method," Carbon, vol. 37, pp. 1449-1453, 1999.

[6] M. Stancu, G. Ruxanda, D. Ciuparu, and A. Dinescu, "Purification of multiwall carbon nanotubes obtained by AC arc discharge method.Optoelectronics and Advanced Materials, vol. R5( 8), pp. 846-850, 2011.

[7] S.J. Tans, M. H. Devoret, H. Dai, A. Thess, R. E. Smalley, L. J. Georliga, and C. Dekker, "Individual single-wall carbon nanotubes as quantum wires,"Nature, vol.386, pp. 474-477, 1997.

[8] S. J. Tans, R. M. Verschueren, and C. Dekker, "Room temperature transistor based on a single carbon nanotube,"Nature, vol. 393, pp. 49-52, 1998

[9] P. L. McEuen, M. S. Fuhrer, andH. Park."Single-Walled Carbon Nanotube Electronics," IEEE Transactions on Nanotechnolog, vol.1(1), pp. 78-85, 2002.

[10] N. Hamada, S. Sawada, and A. Oshiyama, "New OneDimensional Conductors: Graphitic Microtubules," Phys. Rev. Lett., vol. 68, pp. 1579-1581, 1992.

[11] C. Garau, A. Frontera, D. Quinonero, A. Costa, P. Ballester, P. M. Dey, " Lithium diffusion in single-walled carbon nanotubes: a theoretical study," Chem. Phys. Lett., vol.374, pp. 548-555, 2003.

[12] H. Dai, J. H. Hafner, A. G. Rinzler, D. T. Colbert, and R. E. Smalley, "Nanotubes as Nanoprobes in Scanning Probe Microscopy," Nature, vol. 384,pp. 147, 1996.

[13] K. T. Lau, ChongGu and D. Hui, "A Critical Review on Nanotube and Nanotube/nanoclayRelated Polymer Composite Materials," Composites, vol. B 37, pp. 425-436, 2002.

[14] J. H. Ahn, G. X. Wang, H.K. Liu,and S. X. Dou, "Nanoparticle-dispersed PEO polymer electrolytes for $\mathrm{Li}$ batteries," J. Power Sour, vol.119-121, pp.422-426, 2003.

[15] W. A. de Heer, A. Chatelain, and D. Ugarte, "A Carbon Nanotube Field-Emission Electron Source," Science, vol. 270, pp. 1179-1180, 1995. 
[16] R. Martel, T. Schmidt, H. R. Shea, T. Hertel, and Ph. Avouris, "Single- and multi-wall carbon nanotube field-effect transistors," Appl. Phys. Lett., vol. 73, pp. 2447-2449, 1998.

[17] A. K. Alexander, S. B. Lee, Z. Mei, R. H. Baughman, A. A. Zakhidov, "Electron field emission from transparent multiwalled carbon nanotube sheets for inverted field emission displays," Carbon, vol.48, pp. 41-46, 2010.

[18] F. Kun, O. Yildiz, H. Bhanushali, Y. Wang, K. Stano, L. Xue ,X. Zhang, and P. D. Bradford, "Aligned Carbon Nanotube-Silicon Sheets: A NovelNano-architecture for Flexible Lithium IonBattery Electrodes," Adv. Mate, pp. 1-6, 2013.

[19] A. Jensen, J. R. Hauptmann, J. Nygrd, J. Sadowski, andP. E. Lindelof, "Hybrid Devices from Single Wall Carbon Nanotubes Epitaxially Grown into a Semiconductor Heterostructure," Nano Lett.4 (2), pp. 349-352, 2004.

[20] Z. P. Huang, D. Z. Wang, J. G. Wen, M. Sennett, H. Gibson, and Z. F. Ren, "Effect of nickel, iron and cobalt on growth of aligned carbon nanotubes," Appl. Phys.vol. A74, pp. 387, 2002.

[21] K. Hernadi, A. Fonseca, J. B. Nagy, D. Bernaerts, and A. A. Lucas, "Fe-Catalyzed Carbon Nanotubes Formation," Carbon, vol. 34 (10), pp. 1249, 1996.

[22] L. Shao, G. Tobias, C. G. Salzmann, B. Ballesteros, S. You Hong, A. Crossley, B. G. Davis, and M. L. H. Green, "Removal of amorphous carbon for the efficient sidewall functionalisation of single-walled carbon nanotubes," Chem. Commun., pp. 5090-5092, 2007.

[23] C. Amente, and K. Dharamvir, "Thermally Agitated Self Assembled Carbon Nanotubes and the Scenario of
ExtrinsicDefects,” W. J. Nano Sci. and Eng., vol. 5, pp.17-25, 2015.

[24] P. Mahanandia, K. K.Nanda, V. Prasad, and S. V. Subramanyam, "Synthesis and characterization of carbon nanoribbons and single crystal iron filled carbon nanotubes," Mat. Research Bulletin, vol.43, pp. 3252-3262, 2008.

[25] W. Kici'nski, M. Szala, andM. Nita, "Structurally tailored carbon xerogels produced through a sol-gel process in a water-methanol-inorganic salt solution," J. Sol-Gel. Sci. Technol, vol. 58, pp. 102, 2011.

[26] P. Scherrer, "Bestimmung der Größe und der innerenStruktur von Kolloidteilchen Mittels Röntgenstrahlen, Nachrichten von der Gesellschaft der Wissenschaften, Gttingen," Mathematisch-Physikalische Klasse, vol. 2, pp. 98-100, 1918.

[27] N. Grobert, "Carbon nanotubes becoming clean," Materials today, vol. 10, pp. 28-35, 2007.

[28] C. Journet, W. K. Maser, P. Bernier, A. Loiseau,M. Lamy de laChapelle, S. Lefrant, P. Deniard, R. Lee, J. E. Fischer, "Large-scale production of single-walled carbon nanotubes by the electric-arc technique," Nature, vol. 388, pp. 756-757, 1997.

[29] T. Dietl, H. Ohno, F. Matsukura, J. Cibert, and D. Ferrand, "Zener model description of ferromagnetism in zinc blende magnetic semiconductors," Science, vol. 287, pp. 1019-1022, 2000 .

[30] Q. Sun, A. K. Kandalam, Q. Wang, P. Jena, Y. Kawazoe, and M. Marquez, "Effect of Au coating on the magnetic and structural properties of $\mathrm{Fe}$ nanoclusters for use in biomedical applications: A density-functional theory study," Phy. Rev., vol. B73, pp. 134409, 2006. 